\title{
Scanning electron microscopy analysis of erythrocytes in thromboembolic ischemic stroke
}

\author{
A.C. Swanepoel and E. Pretorius \\ Department of Physiology, School of Medicine, Faculty of Health Sciences, University of Pretoria, Pretoria, \\ South Africa
}

Correspondence:

E. Pretorius, Department of Physiology, Faculty of Health Sciences, University of Pretoria, Private Bag x323, Arcadia 0007, South Africa.

Tel.: +2712420 2864;

Fax: +27 124204482

E-mail: resia.pretorius@up.ac.za

\section{Summary}

Introduction: Erythrocytes play an important role in hemostasis and disease conditions. During ischemic stroke, erythrocytes undergo oxidative and proteolytic changes resulting in a changed cellular rheology.

Methods: Blood samples were obtained from controls and thromboembolic ischemic stroke patients (within $48 \mathrm{~h}$ of stroke). The ultrastructure of erythrocytes was compared, using a scanning electron microscope (SEM). Abnormal morphology included codocytes, knizocytes, stomatocytes, and echinocytes. Percentage of abnormal cells was calculated, and the analyses were performed using the statistical program NCSS with the level of significance set at 0.05. A $t$-test was carried out to compare the data from the erythrocyte counts of stroke patients with that of the control subjects.

Results: Ultrastructural SEM results showed that there are a large percentage of erythrocytes in healthy individuals that do not have a typical discoid shape, when studying the cells using a high magnification electron microscope. Furthermore, analysis showed that variation in shape is so subtle that it is not clearly visible using a typical light microscopy blood smear analysis. Thromboembolic ischemic stroke patients presented with a significant amount of erythrocytes with abnormal morphology.

Conclusion: We suggest that in healthy individuals, a typical smear would contain several nondiscoid-shaped erythrocytes, only clearly visible at high magnification. However, thromboembolic ischemic stroke does significantly impact erythorcyte shape, and this change in morphology may result in an impaired microcirculation, as well as impaired oxygen carrying capacity. This changed morphology may further complicate the restoring of homeostasis caused by acute thromboembolic stroke.

Keywords: Thromboembolic stroke, erythrocytes, scanning electron microscopy 


\section{Introduction}

Erythrocytes play an important role in hemostasis and disease conditions. It is well-known that they have a particular and very well-defined shape when studying them under the light microscope. The discipline of the flow as well as the deformation of blood is referred to as blood rheology. The importance of blood rheology for the clinical application concerns the two major components of circulatory resistance, namely the vascular component and the rheological component. In the large blood vessels, it is bulk flow and the viscosity of the blood (consisting of the concentration of erythrocytes as well as the viscosity of the plasma) that add to the rheology of the blood. The deformability and aggregation of the erythrocytes do not play such a big role in macrocirculation. However, when the erythrocytes travel through narrow capillaries, these cells need to deform to enable them to pass through. Thus, in microcirculation, the resistance flow is mainly influenced by the ability of individual cells to deform, also referred to as cellular rheology (Stuart \& Nash, 1990).

Cellular rheology also has an effect on the survival time of the cell in circulation. The structure of the cell, which includes the geometry of the cell, membrane properties, and the viscosity of the cytoplasm, is directly associated with cell's ability to deform. Because certain hematological illnesses are connected to structural abnormalities, the blood flow in the microcirculation, as well as the lifespan of the erythrocyte, will be involved (Stuart \& Nash, 1990).

The rheological characteristics of erythrocytes can be negatively influenced by various factors. Extracellular factors include the concentration of cholesterol, fibrinogen, and gammaglobulins found in the plasma. Factors connected with the structural conformation of the cell membrane as well as the intracellular levels of ATP, which are inversely proportional to the cytosolic concentration of calcium, also impair the rheology of the erythrocyte (Forconi et al., 1990; Forconi, Guerrini \& Turchetti, 1992).

In ischemic stroke patients, the number of leukocytes is elevated and they are also activated (Santos-Silva et al., 2002). When these leukocytes are activated, they release activation products that affect adjacent cells, including the erythrocytes. As the erythrocytes undergo oxidative and proteolytic changes resulting from their contact with the activation products, they lose their flexibility and build up in the narrow blood vessels of the microcirculation. Blood flow is decreased, and this results in a prolonged period of contact between the activation products accumulating at the blockage and the adjoining cells (Santos-Silva et al., 2002). Erythrocyte sedimentation can then occur, and Chamorro et al. (1995) implicated an elevated erythrocyte sedimentation rate in the critical damage caused by stroke.

Pretorius et al. (2011) showed that in this stroke population, a changed coagulation process influenced the ultrastructure of the fibrin networks that plays a crucial role in hemostasis, and the fibrin networks were markedly changed compared to that of control subjects. The current research investigates the effect of acute thromboembolic ischemic stroke on erythrocyte ultrastructure and changes of the external surface of the erythrocytes, using a quantitative scanning electron microscopy analysis. 


\section{Materials and methods}

A total number of 20 thromboembolic ischemic stroke patients were included in this study, consisting of five men and 15 women. The biological parameters as well as the clinical severity of this patient population were homogenous, and the mean representative age of the population group was 64 years of age. All patients underwent magnetic resonance brain scanning to exclude all other causes and confirm the thromboembolic ischemic stroke. Within $48 \mathrm{~h}$ after admission to hospital, $5 \mathrm{~mL}$ of blood was drawn from each stroke patient into a citrate tube. In the current study, 10 healthy age-matched individuals were used as controls. These individuals were not using any medication; they do not smoke and they did not suffer from any ailment associated with erythrocyte modification including latent iron deficiency.

\section{Preparation of blood pellet for SEM}

Once venous blood was drawn from each subject, the blood was immediately prepared at room temperature for microscopic analysis upon arrival at the laboratory. After centrifuging the citrated blood at $1250 \mathrm{~g}, 400 \mu \mathrm{L}$ of the thick blood pellet was transferred to a glass vial and immediately fixed in a mixture of phosphate-buffered saline, distilled water, $2.5 \%$ gluteraldehyde, and formaldehyde in the ratio of $5: 3: 1: 1$, respectively. After $30 \mathrm{~min}$ of fixation, the sample was rinsed thrice in $0.075 \mathrm{~m}$ sodium potassium phosphate buffer $(\mathrm{pH}=7.4)$ for $5 \mathrm{~min}$ before being placed in secondary fixative and a $1 \%$ osmium tetraoxide solution, for $30 \mathrm{~min}$. Following secondary fixation, the sample was rinsed three times in $0.075 \mathrm{~m}$ sodium potassium phosphate buffer $(\mathrm{pH}=7.4)$ for $5 \mathrm{~min}$. Subsequently, the sample was dehydrated in 30\%, 50\%, 70\%, 90\%, and three changes of $100 \%$ ethanol.

The sample remained in the glass vial for the whole procedure. After each step, the sample was centrifuged and the particular chemical drawn off and discarded.

The scanning electron microscope (SEM) procedures were completed by drying the samples with hexamethyldisilazane (HMDS) for $30 \mathrm{~min}$. After the $30 \mathrm{~min}, 200 \mu \mathrm{L}$ of the sample and HMDS were placed on a cover slip. The cover slip was left to air dry and then coated with carbon. The sample was examined with a Zeiss Ultra plus FEG SEM (Carl Zeiss Microscopy, Europe, Germany).

For statistical analysis of the number of abnormal erythrocytes in the blood, 10 SEM micrographs of 20 stroke patients and 10 healthy individuals were examined. SEM samples from each individual were thoroughly examined to establish the overall morphology of the erythrocytes. Then, 10 SEM micrographs of each individual were taken at low magnification, and up to a 100 erythrocytes were counted per digitized photograph of the micrographs. Each photograph was independently examined and the morphology categorized as either normal or abnormal. The abnormal morphology included codocytes (target cells), knizocytes, stomatocytes, and echinocytes. Percentage of abnormal cells present was calculated, and analysis was performed using the statistical program NCSS with the level of significance set at 0.05. A $t$-test was carried out to compare the data from the erythrocyte counts of stroke patients with that of the control subjects. 


\section{Results and discussion}

Typically, in healthy individuals, there are a percentage of abnormal erythrocytes. A literature search did not suggest that the percentage of changed erythrocytes in healthy individuals has previously been determined using a SEM investigation. However, literature has suggested that there may be five different subshapes, and this was shown by using light microscopy from a blood smear. According to a light microscopy study in $1997,55 \%$ of the erythrocytes were that of the most deformable, bowl-shaped morphology; $44 \%$ were that of the more rigid discocytes; and only $1 \%$ of erythrocytes had altered morphology, mainly that of echinocytes and knizocytes (Turchetti, 1997).

Erythrocyte shape changes can be categorized as follows:

- A discocyte has a discoid or biconcave shape with a shallow, round depression in the center of the cell. This is typical erythrocyte morphology.

- A codocyte is bell-shaped with a considerable reduction in thickness; a single concavity is present, with an extruded opposite side or flattened ridge around the elevated central area of the cell.

- A knizocyte appears pinched in the center, and this area can be either elevated or elongated; there may be two or more invaginations on the membrane.

- A stomatocyte is cup-shaped with a slit-like central area or stoma; these cells have a swollen cell periphery.

- - An echinocyte shows regularly spaced short projections or spicules; the cell periphery is deformed.

According to our investigation, it is very difficult to determine a changed morphology, by doing a light microscopy analysis using a blood smear. This is particularly due to that fact that the cells are flattened in the process of making the smears and also the low magnification capabilities of the light microscope. With this microscope, the fine surface morphology is not clearly visible. In the current analysis, a method was followed whereby the blood was dropped onto a cover slip, with as little as possible physical pressure that might encourage induced deformities.

Although previous light microscopy analysis showed $1 \%$ as an expected standard abnormal percentage of erythrocytes for healthy individuals, the current SEM analysis displayed a higher percentage. Graph 1 shows the percentage normal and abnormal erythrocytes of the control population and the stroke patient population. Dark gray $=$ percentage normal erythrocytes, Light gray $=$ percentage abnormal erythrocytes. 


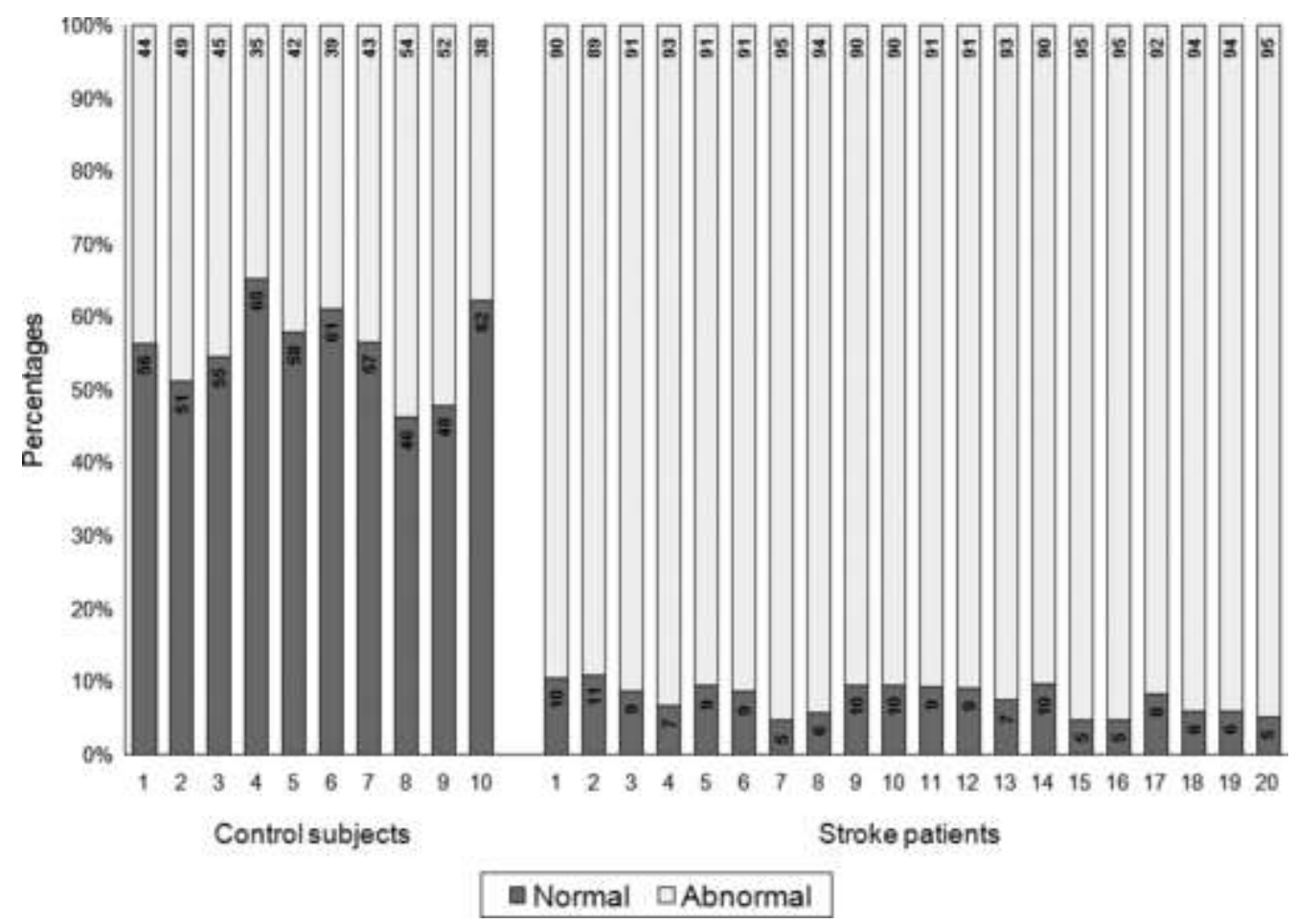

Figure Graph 1. Percentage normal and abnormal erythrocytes of the control population and the stroke patient population. Dark gray = percentage normal erythrocytes, Light gray = percentage abnormal erythrocytes.

Figure 1a shows a micrograph of a healthy individual, depicting the typical discocyte morphology of erythrocytes. In this micrograph, it is clear that minor differences, only visible using a high magnification, would probably go unnoted in a light microscopy blood smear investigation. Figure 2 shows individual erythrocytes indicating the typical variation in erythrocyte morphology.
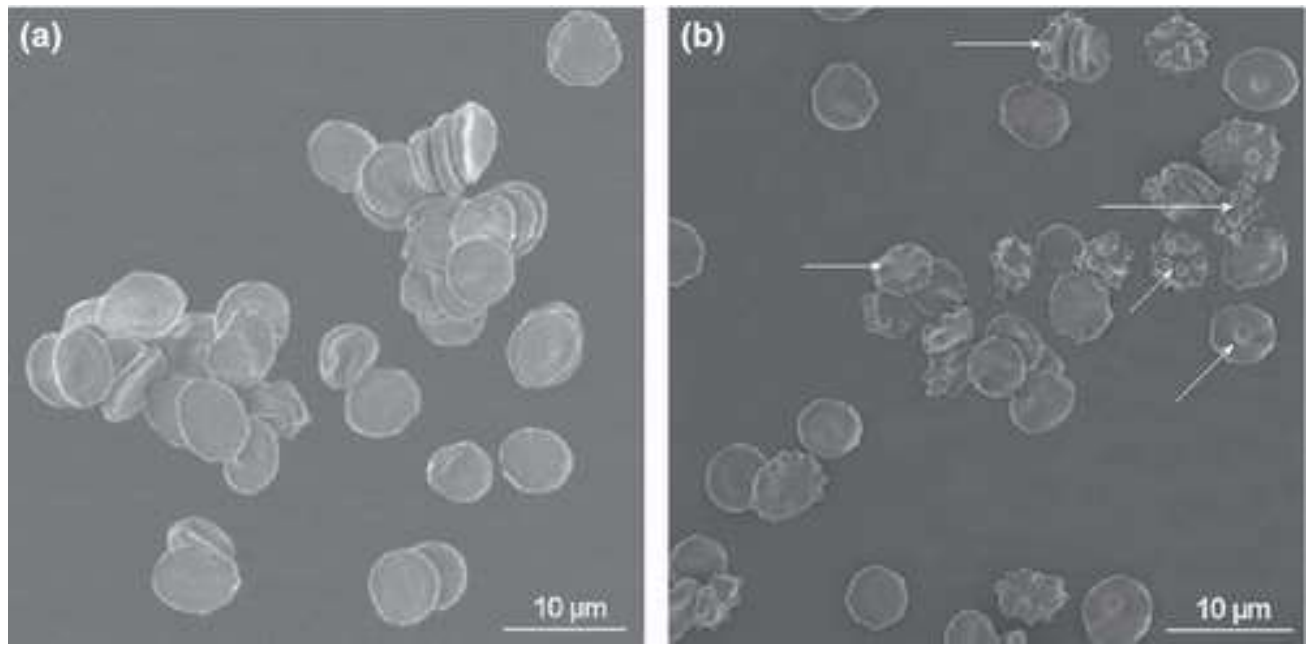

Figure 1. Scanning electron micrograph of variation in erythrocyte morphology. $a=$ Typical clusters of erythrocytes found in the blood of controls, $b=$ Altered morphology exhibited in the blood of the thromboembolic ischemic stroke patients. Arrows = variation in morphology. 

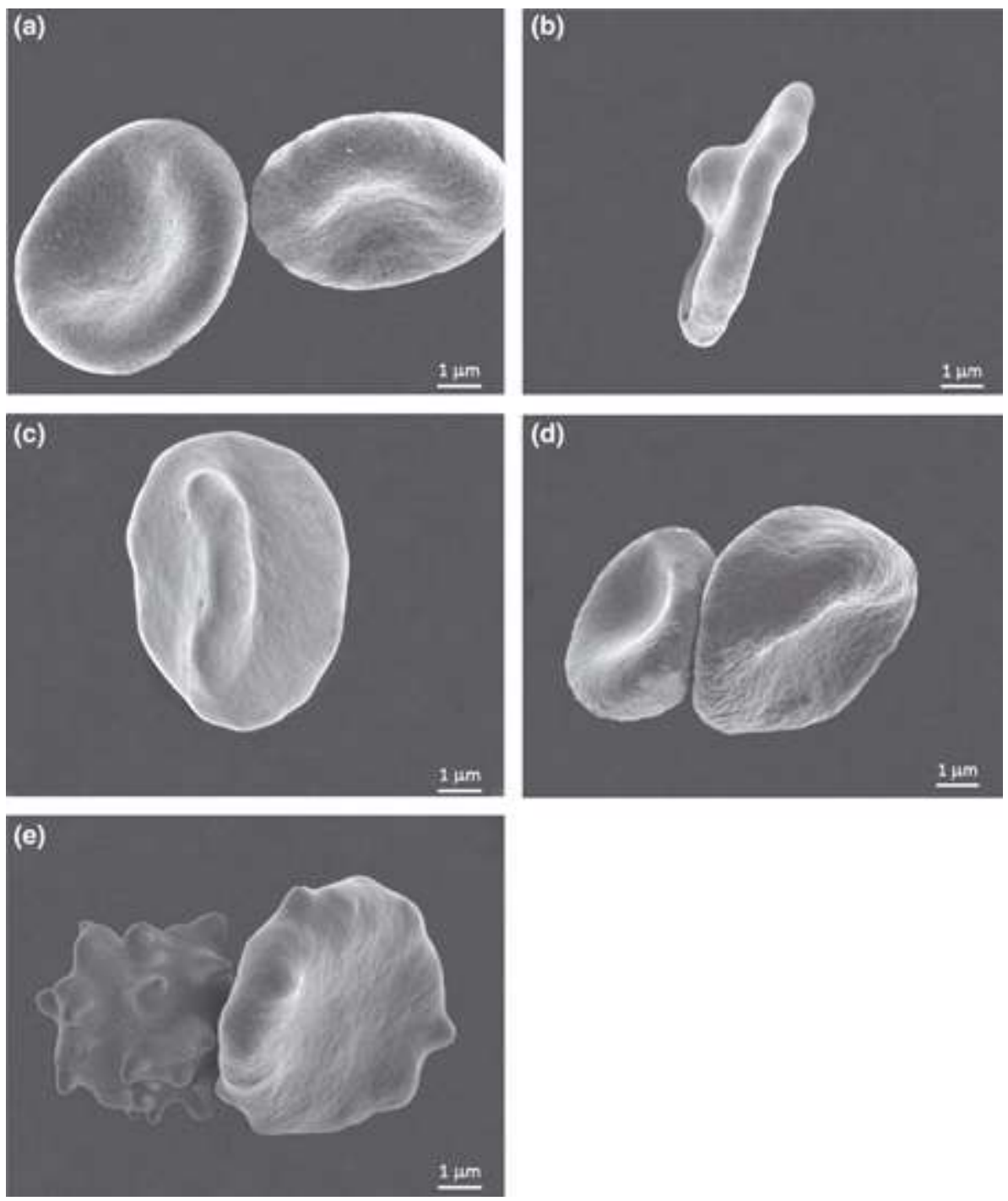

Figure 2. Scanning electron micrographs of individual variation in erythrocyte morphology. $a=$ Discocytes (normal morphology), $\mathrm{b}=$ Codocyte, $\mathrm{c}=$ Knizocyte, $\mathrm{d}=$ Stomatocyte, $\mathrm{e}=$ Echinocytes.

In the current sample of stroke patients, the average percentage of abnormal erythrocytes was $92 \%$, and a $t$-test showed that stroke patients had a significantly larger percentage of abnormal erythrocytes compared to the levels in healthy individuals.

These abnormalities are representative of the four abnormal erythrocyte shapes as can be seen in Figure 2. The erythrocyte morphology presented as follows: $8 \%$ of erythrocytes had a normal, discoid appearance, $80 \%$ of the erythrocytes had the appearance of echinocytes while $3 \%, 4 \%$, and $5 \%$ of erythrocytes displayed ultrastructural abnormalities associated with knizocytes, codocytes, and stomatocytes, respectively. Thus, in the stroke patients, the echinocyte morphology was the predominant ultrastructure observed.

The red blood cell count was within the normal range of $3.8-5.2 \times 10^{12} / \mathrm{L}$ for $67 \%$ of the stroke patients. The other-third of the patients had decreased red blood cell counts. Only $47 \%$ of the patients had increased leukocyte counts above the normal value of $4-10 \times 10^{9} / \mathrm{L}$, as previously implicated by Santos-Silva et al. (2002). 
Figure $1 \mathrm{~b}$ show the variation in erythrocyte morphology of typical clusters of erythrocytes found in the blood of thromboembolic ischemic stroke patients. If we compare this view to that of the healthy individuals (Figure 1a), a visible difference is noted.

The statistical analysis, with utilization of a $t$-test, showed that the stroke patients had a significantly larger percentage of abnormal erythrocytes compared to that found in healthy control individuals. In the current research, it was found that $92 \%$ of the erythrocytes in thromboembolic ischemic stroke did not show the typical discocyte morphology. In healthy individuals less than half of the erythrocytes displayed abnormal morphology (44\%).

This change in morphology noted in the current research may be due to changes in rheology because of the pathology. Numerous studies have confirmed the erythrocyte's reduced ability to deform in ischemic pathological events (Forconi, Guerrini \& Ravelli, 1979; Forconi et al., 1983; Forconi, 1988; Hung et al., 1991). From a membrane mechanics viewpoint, the variation between the two membrane leaflets, concerning area as well as tension, could instigate the advance of a considerable spontaneous curvature of the lipid bilayer (Petrov, 1999).

Sheetz and Singer (1974) as well as Evans (1974) proposed a model to possibly explain the transformation of discocytes to either stomatocytes or echinocytes. According to their bilayer-coupled hypothesis, convex structures, such as the distinguished spicules of echinocytes, form when any factor causes the outer membrane leaflet to expand relative to the inner leaflet. In contrast, cavities are formed when inner membrane leaflet expand relative to the outer leaflet, and the extra area has to be accommodated, leading to the formation of stomatocytes (Evans, 1974; Sheetz \& Singer 1974).

Shear stress may have an effect on the ultrastructure of the erythrocytes. Shear stress refers to the force of friction from a fluid acting on a body in the path of that fluid [Shear Stress, 2006. Available at:

http://www.fsl.orst.edu/geowater/FX3/help/8_Hydraulic_Reference/Shear_Stress.htm (accessed 4 January 2011)]. Shear stress can thus be interpreted as the effect that plasma friction force has on blood cell, including erythrocytes. In macrocirculation, as well as microcirculation exists a significant rheological occurrence - the erythrocytes membrane continuously rotates around the liquid content of the cell. This causes the external shear stress to be transferred to the cytoplasm through the cell membrane. The cytoplasm surges in a circular motion within the cell as the membranes' lipid-protein planes alternate sliding forward and backward. The rheological behavior of red blood cells is thus controlled by the microviscous attributes of the cell membrane and cytoplasm (Dormandy, 1983; Stoltz, 1983; Forconi, Pieragalli \& Guerrini, 1987).

Leverett and coworkers found that the variation in the force and exposure time of shear stress significantly influence the amount of damage to the erythrocytes in circulation. They indicated that little damage occurs when both the force and exposure time of shear stress is low, because the damage is subject to the effects of solid surface interaction. Hemolysis dominantly occurs when the force of the shear stress is very high over an extended period of time (Leverett et al., 1972).

White blood cells can also indirectly cause alterations in the cell membrane. When white blood cells or leukocytes are activated, they release proteases and oxygen metabolites which in turn have proteolytic and oxidative effects on the membrane of the erythrocytes. These 
membrane alterations lead to destruction of the erythrocyte membrane (Santos-Silva et al., 2002).

\section{Conclusion}

The current study shows that there is a large percentage of erythrocytes in healthy individuals that do not have a typical 'perfect' discoid shape, when studying the cells using a high magnification electron microscope. Typically, five different shapes can be identified, and this is well described in the literature (Howard \& Hamilton, 1999). Interestingly, we found that the variation in shape is so subtle that it is not clearly visible using a typical light microscopy blood smear analysis. We therefore suggest that in healthy individuals, a typical smear would contain several nondiscoid-shaped erythrocytes. However, disease does impact the shape.

Literature suggests that in several pathologies, the morphology of the erythrocyte is changed, resulting in serious impairment of the flow of erythrocytes in the microcirculation (Stoltz, 1983; Forconi, Pieragalli \& Guerrini, 1987). In the current study, thromboembolic ischemic stroke patients presented with a significant amount of erythrocytes with abnormal morphology that include codocytes, knizocytes, stomatocytes, and echinocytes. This change in morphology may result in an impaired microcirculation, as well as impaired oxygen carrying capacity, which may further complicate the restoring of homeostasis caused by acute thromboembolic stroke.

This study focused on the consequence of stroke on erythrocyte morphology within $48 \mathrm{~h}$ after a stroke. We are currently investigating whether this altered morphology persists in the stroke patients by doing a follow-up study of stroke patients, 3 months after the ischemic event. Other diseases associated with hyperoxydative stress should be considered for future studies. This will expand the understanding of disease conditions and also supply a basis for comparison of the etiology of various diseases.

\section{References}

Chamorro A.M., Vila N.M., Ascaso C.P., Saiz A.M., Montalvo J.M., Alonso P.M. \& Tolosa E.M. (1995) Early prediction of stroke severity: role of the erythrocyte sedimentation rate. Stroke 26, 573-576.

Dormandy J.A. (1983) Red Cell Deformability and Filterability. Martinus Nijhoff Publishers, Boston.

Evans E.A. (1974) Bending resistance and chemically induced moments in membrane bilayers. Biophysical Journal 14, 923-931.

Forconi S. (1988) Regional ischemia and hyperviscosity. Clinical Hemorheology 8, 321-339.

Forconi S., Guerrini M. \& Ravelli P. (1979) Arterial and venous blood viscosity in ischemic lower limbs in patients affected by peripheral obliterative arterial disease. Journal of Cardiovascular Surgery 20, 379-384. 
Forconi S., Guerrini M. \& Turchetti V. (1992) Local haemorheological changes and intracellular erythrocyte $\mathrm{Ca}++$ content in exercising ischaemic limb. Clinical Hemorheology 12, 527-534.

Forconi S., Pieragalli D. \& Guerrini M. (1987) Primary and secondary blood hyperviscosity syndromes, and syndromes associated with blood hyperviscosity. Drugs 33(Suppl 2), 19-26.

Forconi S., Guerrini M., Pieragalli D., Acciavatti A., Del Bigo C., Galigani C. \& Di Perri T. (1983) Hemorrheological changes in ischemic heart disease. La Ricerca in Clinica e in Laboratorio 13(Suppl 3), 195-208.

Forconi S., Guerrini M., Cappelli R., Sani P. \& Bicchi Furesi M.L. (1990) Changes of blood viscosity due to the variations of blood flow dynamics in man. Revista Portuguesa de Hemorreologia 3, 217-233.

Howard M.R. \& Hamilton P.J. (1999) Haematology: An Illustrated Colour Text. Churchill Livingston, Edinburgh.

Hung T.C., Pham S., Steed D.L., Webster M.W. \& Butter D.B. (1991) Alterations in erythrocyte rheology in patients with severe peripheral vascular disease: 1 . Cell volume dependence of erythrocyte rigidity. Angiology 42, 210-217.

Leverett L.B., Hellums J.D., Alfrey C.P. \& Lynch E.C. (1972) Red blood cell damage by Shear Stress. Biophysical Journal 12, 257-273.

Petrov A.G. (1999) The Lyotropic State of Matter: Molecular Physics and Living Matter Physics. Gordon \& Breach, Amsterdam.

Pretorius E., Swanepoel A. C., Oberholzer H..M., van der Spuy W.J., Duim W. \& Wessels P.F. (2011) A descriptive investigation of the ultrastructure of fibrin networks in thromboembolic ischemic stroke. Journal of Thrombosis and Thrombolysis 31, 507-513.

Santos-Silva A., Rebelo I., Castro E., Belo L., Catarino C., Monteiro I., Almeida M.D. \& Quintanilha A. (2002) Erythrocyte damage and leukocyte activation in ischemic stroke. Clinica Chimica Acta 320, 29-35.

Sheetz M.P. \& Singer S.J. (1974) Biological membranes as bilayer couples. A molecular mechanism of drug erythrocyte interactions. Proceedings of the National Academy of Sciences of the United States of America 71, 4457-4461.

Stoltz J.F. (1983) Red blood cell microrheology (clinical and pharmacological applications). La Ricerca in Clinica e in Laboratorio 13(Suppl 3), 53-70.

Stuart J. \& Nash G.B. (1990) Red cell deformability and haematological disorders. Blood Reviews 4, 141-147.

Turchetti V. (1997) Variations of erythrocyte morphology in different pathologies. Clinical Hemorheology and Microcirculation 17, 209-215. 